\title{
Raman scattering by LO phonon-plasmon coupled modes in $n$-type InP
}

\author{
L. Artús, R. Cuscó, and J. Ibáñez \\ Institut Jaume Almera, Consell Superior d'Investigacions Científiques (CSIC), Lluís Solé i Sabarís s.n., 08028 Barcelona, Spain \\ N. Blanco and G. González-Díaz \\ Departamento de Física Aplicada III, Facultad de Físicas, Universidad Complutense, 28040 Madrid, Spain
}

(Received 26 February 1999)

\begin{abstract}
We have studied LO phonon-plasmon coupled modes by means of Raman scattering in $n$-InP for carrier densities between $6 \times 10^{16}$ and $1 \times 10^{19} \mathrm{~cm}^{-3}$. A line-shape theory based on the Lindhard-Mermin dielectric function that takes into account the nonparabolicity of the InP conduction band as well as temperature and finite wave-vector effects is used to fit the Raman spectra and extract accurate values of the electron density. The results obtained from the Lindhard-Mermin model are compared with the charge density determinations based on the Drude and the hydrodynamical models, and the approximations involved in these models are discussed. [S0163-1829(99)10431-4]
\end{abstract}

\section{INTRODUCTION}

Numerous studies have been reported on light scattering by free carriers in zinc-blende semiconductors, ${ }^{1}$ and different models have been developed to analyze the coupling between longitudinal optical phonons and plasmons. However, only a few reports are focused on the study of the LOplasmon coupling in doped InP crystals. The first experimental observation of LO phonon-plasmon coupled modes (LOPCM's) in $n$-InP by means of Raman scattering was reported by Zemski et al. ${ }^{2}$ on a single $n$-InP sample, for which a carrier concentration of $1.4 \times 10^{17} \mathrm{~cm}^{-3}$ was obtained from the $L^{+}$coupled-mode frequency using a Drude model. Single particle and collective excitations of free electrons were studied near the $E_{0}+\Delta_{0}$ resonance on a single $n$-InP sample with a carrier concentration of $1.3 \times 10^{18} \mathrm{~cm}^{-3},{ }^{3}$ and the LOPCM's were discussed on the basis of a Drude model. An LOPCM study was also carried out on S-doped InP samples with carrier densities in the $9 \times 10^{16}-4 \times 10^{18} \mathrm{~cm}^{-3}$ range, ${ }^{4}$ in which the hydrodynamical theory was used to determine the carrier concentration from the Raman spectra. Raman LOPCM line shapes on $n$-type InP with three different carrier concentrations, $2 \times 10^{17}, 9 \times 10^{17}$, and $8.6 \times 10^{18}$ $\mathrm{cm}^{-3}$, were later analyzed utilizing a power expansion of the Lindhard-Mermin dielectric function for zero temperature. ${ }^{5}$ A work carried out by Bairamov et al. ${ }^{6}$ on Sn- and S-doped InP reported the observation by means of Raman scattering of $L^{+}$modes only in two of the four samples measured whereas the $L^{-}$modes were detected in all samples. The Raman results presented in that paper, as well as their analysis based on a Drude model, show significant discrepancies with the results of the present work. A Raman-scattering study of $n$-type InP obtained by different growth techniques and dopants in a wide carrier density range $\left(2.6 \times 10^{16}\right.$ $3.7 \times 10^{19} \mathrm{~cm}^{-3}$ ) was later reported, but the carrier concentration was also determined by fitting a Drude model. ${ }^{7}$ LOPCM's were also observed in Raman-scattering measurements at $15 \mathrm{~K}$ on a single sample of Si-implanted InP. ${ }^{8} \mathrm{~A}$ broad peak centered at about $460 \mathrm{~cm}^{-1}$ was assigned to an $L^{+}$mode, and a carrier concentration of $1.5 \times 10^{18} \mathrm{~cm}^{-3}$ was estimated again by applying a Drude model. Recently, it has been shown that a crossover of zone center $(\Gamma)$ and zone boundary $(X)$ conduction-band edges takes place in heavily doped $n$-InP under hydrostatic pressure, which leads to a charge transfer from the $\Gamma$ to the $X$ valley, where the electrons become bound to the $X$-related donors. The corresponding reduction of free-electron density was observed as a decrease of the $L^{+}$coupled-mode frequency, and a LindhardMermin model was applied to determine the free-electron density variations with pressure. ${ }^{9}$

The Drude model and the hydrodynamic theory have been widely used to obtain carrier concentrations from the analysis of Raman scattering by LOPCM's in semiconductors. The Drude model approach used to analyze LOPCM's in most of the papers on InP listed above $\mathrm{e}^{2,3,6-8}$ has several shortcomings: (1) The dependence of the electronic susceptibility on wave vector is ignored. (2) A parabolic conduction band is assumed. (3) The temperature at which the experiments are carried out is not taken into account. On the other hand, the hydrodynamical theory ${ }^{10}$ incorporates the $q$ dependence of the electronic susceptibility, but parabolic conduction bands and degenerate conditions are usually assumed in the calculations and hence nonparabolicity and temperature effects are neglected. ${ }^{4}$ In Ref. 5, the Raman spectra were fitted using the Lindhard-Mermin dielectric function, but zero temperature was assumed in order to derive an analytical expression for the small- $q$ electronic susceptibility that included a nonparabolicity correction. The theoretical Raman line shapes were fitted only to the $L^{+}$mode, even for the Raman spectrum of the only sample in which the $L^{-}$mode was detected, and the photoexcited charge was not taken into account in the determination of the electron density in the low-doping sample.

Recently, we have shown that the photoexcited charge has measurable effects on the Raman-scattering spectra of semiconductor and semi-insulating InP. ${ }^{11}$ Consequently, the presence of photoexcited carriers has to be considered in the analysis of light scattering by free carriers in lightly doped InP samples. In our previous work, ${ }^{11}$ we applied a LindhardMermin model that included the contributions to the electric susceptibility of electron, heavy-hole, and light-hole intraband transitions, as well as interband heavy-hole-light-hole 
TABLE I. List of the $n$-type InP samples studied in this work. The Si dopants were introduced by double ion-beam implantation at 50 and $150 \mathrm{keV}$. The implantation doses listed in the second and third columns result in a doping profile with a flat region that extends beyond the laser penetration depth. The electron density as determined by Hall measurements $\left(N_{e}^{(\mathrm{Hall})}\right)$, as well as the electron density and damping constant obtained from the line-shape analysis of the Raman spectra using the Lindhard-Mermin model are also listed.

\begin{tabular}{cccccc}
\hline \hline \multicolumn{7}{c}{$\begin{array}{c}\text { Implantation dose } \\
50 \mathrm{keV}\end{array}$} & $\begin{array}{c}150 \mathrm{keV} \\
\left(\mathrm{cm}^{-2}\right)\end{array}$ & $\begin{array}{c}N_{e}^{(\text {Hall })} \\
\left(\mathrm{cm}^{-3}\right)\end{array}$ & $\begin{array}{c}N_{e} \\
\left(\mathrm{~cm}^{-3}\right)\end{array}$ & $\begin{array}{c}\Gamma_{e} \\
\left(\mathrm{~cm}^{-1}\right)\end{array}$ \\
\hline Sample & $6.0 \times 10^{11}$ & $3.4 \times 10^{12}$ & $\approx 7 \times 10^{16}$ & $5.7 \times 10^{16}$ & 80 \\
$B$ & $2.1 \times 10^{12}$ & $1.2 \times 10^{13}$ & $3.5 \times 10^{17}$ & $3.7 \times 10^{17}$ & 90 \\
$C$ & $3.5 \times 10^{12}$ & $1.9 \times 10^{13}$ & $5.7 \times 10^{17}$ & $6.6 \times 10^{17}$ & 92 \\
$D$ & $4.5 \times 10^{12}$ & $2.5 \times 10^{13}$ & $8.4 \times 10^{17}$ & $8.4 \times 10^{17}$ & 94 \\
$E$ & $8.5 \times 10^{12}$ & $4.7 \times 10^{13}$ & $1.4 \times 10^{18}$ & $1.4 \times 10^{18}$ & 110 \\
$F$ & $2.3 \times 10^{13}$ & $1.2 \times 10^{14}$ & $3.7 \times 10^{18}$ & $3.5 \times 10^{18}$ & 136 \\
$G$ & $3.5 \times 10^{13}$ & $1.9 \times 10^{14}$ & $5.1 \times 10^{18}$ & $5.7 \times 10^{18}$ & 223 \\
$H$ & $8.9 \times 10^{13}$ & $5.0 \times 10^{14}$ & $1.0 \times 10^{19}$ & $1.1 \times 10^{19}$ & 330 \\
\hline \hline
\end{tabular}

transitions. Considering the typical values of photoexcited carrier densities, in that paper we assumed MaxwellBoltzmann velocity distributions and parabolic band dispersions.

The aim of the present paper is to study light scattering by free carriers in $n$-type InP for a wide range of carrier concentrations, from about $6 \times 10^{16}$ to $1 \times 10^{19} \mathrm{~cm}^{-3}$. Accurate values of electron density in $n$-InP are obtained from the analysis of the LOPCM line shapes using an extension of the model that was already applied successfully to the photoexcited plasma. The extended model includes the Fermi distribution function and the nonparabolicity effects in the calculation of the electronic susceptibility, and thus provides an accurate description of the electron system over the whole range of electron densities that we study. Also, a correction to account for photogenerated carriers has been made on the sample with the lowest doping level, in accordance with our previous results on photoexcited plasmons. This correction, which for low-doping samples can be a substantial fraction of the charge that gives rise to the LOPCM's, was not subtracted in any of the previous works, which therefore overestimate the charge density in samples with low doping levels.

Most of the charge-density determinations from LOPCM's in semiconductors that can be found in the literature rely either on fits of the Drude or the hydrodynamical model to the $L^{+}$coupled modes only. For InP in particular, no line-shape fit to the $L^{-}$modes has been published. In the present work, the use of the Lindhard-Mermin model allows us to carry out accurate line-shape fits to the $L^{+}$and $L^{-}$ peaks simultaneously.

The Raman measurements on $n$-type InP show that the $L^{+}$coupled mode is very sensitive to carrier concentration and thus large frequency shifts of the $L^{+}$peak are observed within the range of electron densities studied. Therefore, Raman spectroscopy is a powerful, nondestructive technique to determine the electron concentration in $n$-doped $\mathrm{InP}$ with high accuracy if the analysis of the LOPCM's is carried out using a Lindhard-Mermin model that includes band nonparabolicity. The results thus obtained are discussed in comparison with those obtained from other commonly used models, such as the Drude and the hydrodynamical models. The
Drude model, including an electronic damping parameter, is actually applied to determine the carrier density in many analyses of LOPCM's in zinc-blende semiconductors and other compounds being nowadays actively investigated, such as $\mathrm{SiC}$ and $\mathrm{GaN} .{ }^{12-16}$ In the present paper, we show that the carrier concentrations obtained by applying either the Drude or the hydrodynamical model differ substantially from those obtained by applying the Lindhard-Mermin model. For a certain range of carrier densities, the Drude model may accidentally yield only small differences in relation to the LindhardMermin model, but this is due to the partial compensation of different effects that are neglected in the Drude model.

\section{EXPERIMENT}

The $n$-type doped InP samples were obtained by ion-beam implantation of ${ }^{28} \mathrm{Si}^{+}$into (100)-oriented wafers of semiinsulating InP:Fe, grown by Sumitomo using the liquidencapsulated Czochralski method. Double implantations at 50 and $150 \mathrm{keV}$ were carried out in order to produce homogeneous doping profiles over a length larger than the probing depth of the laser light used in the Raman measurements. The implantation doses at these energies required for each overall doping concentration were determined from TRIM (Ref. 17) simulations and are listed in Table I. According to TRIM calculations, these implantations result in approximately flat doping profiles between depths of 60 and $200 \mathrm{~nm}$, where charge-density variations are less than $5 \%$. To avoid channeling effects, the implantations were carried out with the samples tilted $7^{\circ}$ off normal incidence. Dopant activation was achieved by rapid thermal annealing at $875^{\circ} \mathrm{C}$ for $10 \mathrm{~s}$ using an RTP-600 system from MPT Corp., in a graphite susceptor, face down on a $\mathrm{Si}$ wafer in a P-rich atmosphere. Finally, a layer of about $60 \mathrm{~nm}$ was removed from the surface of the samples by chemical etching to obtain an homogeneous doping profile starting at the surface and extending to a depth of $\approx 140 \mathrm{~nm}$. The dopant activation was determined by Hall measurements in the van der Pauw geometry as the quotient between sheet carrier density and implantation dose. The Hall factor was taken as unity. The actual electron concentration in the homogeneously doped layer was calculated from the measured activation assuming the 
doping profile generated by TRIM simulations, and the corresponding values for the different samples are shown in the fourth column of Table I. The Raman measurements were performed on a (100) face using the 528.7-nm line of an $\mathrm{Ar}^{+}$ laser, with a power on the sample of $\approx 100 \mathrm{~mW}$, in a $x(y z) \bar{x}$ backscattering configuration. For this wavelength, using the absorption coefficient of InP ${ }^{18}$ we estimate that just about $10 \%$ of the incident intensity is still acting as probing light at a depth of $110 \mathrm{~nm}$, and consequently, the Raman-scattering measurements are only probing the homogeneously doped layer. The Raman spectra were recorded using a Jobin-Yvon T64000 spectrometer equipped with a charge coupled device detector cooled with liquid nitrogen. The spectra were taken at room temperature using the double subtractive configuration of the spectrometer with $100-\mu \mathrm{m}$ slits. To better resolve the $L^{-}$peak from the TO peak, measurements at $80 \mathrm{~K}$ with the triple additive configuration of the spectrometer and $100-\mu \mathrm{m}$ slits were also performed using a TBT liquid nitrogen cryostat.

\section{THEORETICAL MODEL}

The Raman line-shape analysis of the LOPCM's has been carried out using the fluctuation-dissipation theory developed by Hon and Faust. ${ }^{19}$ With a suitable choice of the free-charge susceptibility, the fluctuation-dissipation formalism has proved to be very powerful to calculate LOPCM line shapes in $n$-type ${ }^{19}$ and $p$-type ${ }^{20,21}$ semiconductors, and also to model photoexcited electron-hole plasmas in semiconductors. ${ }^{11,22}$ In this formalism, taking into account the electro-optic and the deformation-potential scattering mechanisms, the differential Raman cross section for the LOPCM's can be expressed as

$$
\frac{\partial^{2} \sigma}{\partial \omega \partial \Omega} \propto \Im\left\{\frac{-1}{\epsilon(\omega)}\left[\frac{\epsilon_{\infty}}{4 \pi}+2 A \chi_{I}-A^{2} \chi_{I}\left(1+\frac{4 \pi}{\epsilon_{\infty}} \chi_{e}\right)\right]\right\},
$$

where $\epsilon(\omega)=\epsilon_{\infty}+4 \pi\left(\chi_{I}+\chi_{e}\right)$ is the total dielectric function of the electron plasma and

$$
A=\frac{\omega_{\mathrm{TO}}^{2}}{\omega_{\mathrm{LO}}^{2}-\omega_{\mathrm{TO}}^{2}} C .
$$

$C$ is the Faust-Henry coefficient, and $\omega_{\mathrm{TO}}$ and $\omega_{\mathrm{LO}}$ are the zone center transverse and longitudinal optical mode frequencies, respectively. $\chi_{\mathrm{I}}$ is the ionic lattice contribution to the susceptibility, which arises from the dipole moments induced by the longitudinal optical modes, and is given by

$$
\chi_{\mathrm{I}}=\frac{\epsilon_{\infty}}{4 \pi} \frac{\omega_{\mathrm{LO}}^{2}-\omega_{\mathrm{TO}}^{2}}{\omega_{\mathrm{TO}}^{2}-\omega^{2}-i \Gamma_{\mathrm{I}} \omega},
$$

where $\Gamma_{\mathrm{I}}$ is a phenomenological ionic damping constant. $\chi_{e}$ is the wave-vector and frequency-dependent electron-plasma susceptibility, which can be calculated using the LindhardMermin expression ${ }^{23}$

$$
\chi_{e}\left(q, \omega+i \Gamma_{e}\right)=\frac{\left(1+i \Gamma_{e} / \omega\right) \chi_{e}^{L}\left(q, \omega+i \Gamma_{e}\right)}{1+i \Gamma_{e} \chi_{e}^{L}\left(q, \omega+i \Gamma_{e}\right) /\left(\omega \chi_{e}^{L}(q, 0)\right)},
$$

where $\chi_{e}^{L}$ is the Lindhard susceptibility. The LindhardMermin model of the electronic susceptibility includes collision-damping effects in the relaxation-time approximation through the phenomenological damping constant $\Gamma_{e}$ $=1 / \tau$.

In our previous work on photoexcited electron-hole plasmons in InP, ${ }^{11}$ we assumed that the photoexcited carriers were thermalized to a Maxwell-Boltzmann velocity distribution. This was a good approximation as the photoexcitedcarrier density was in the $10^{16}-10^{17} \mathrm{~cm}^{-3}$ range. By contrast, the electron densities in the $n$-doped samples studied in this work cover the $10^{16}-10^{19} \mathrm{~cm}^{-3}$ range, which makes the classical assumption invalid. The electric susceptibility $\chi_{e}^{L}$ has been obtained by numerical evaluation of the Lindhard integral ${ }^{23,24}$

$$
\begin{aligned}
\chi_{e}^{L}(q, \omega)= & \frac{e^{2}}{2 \pi^{3} q^{2}} \int f\left(E_{F}, T, k\right) \\
& \times \frac{E(q+k)-E(k)}{[E(q+k)-E(k)]^{2}-(\hbar \omega)^{2}} d^{3} k,
\end{aligned}
$$

where $f\left(E_{F}, T, k\right)$ is the Fermi distribution function for an electron gas with Fermi energy $E_{F}$ at temperature $T$, and $E(k)$ is the energy dispersion of the InP conduction band. As the samples studied in this work cover a wide range of doping concentrations, neither the classical Maxwellian limit nor the degenerate limit are good approximations to the electron distribution function for the samples with intermediate electron densities, and therefore the numerical evaluation of Eq. (3.5) is necessary.

Besides, at high electron densities, conduction band nonparabolicity has an important effect on plasmon energies. ${ }^{24,25}$ The nonparabolicity of the InP conduction band was taken into account in the calculation through the isotropic, polynomial band dispersion including terms up to $k^{6}$

$$
E(k)=A_{2} k^{2}+A_{4} k^{4}+A_{6} k^{6} .
$$

The coefficients $A_{2}, A_{4}, A_{6}$ were obtained by fitting the polynomial band dispersion to the results of a $14 \times 14 \mathbf{k} \cdot \mathbf{p}$ model, which explicitly includes the interactions among the $\Gamma_{7}$ splitoff valence band, the $\Gamma_{8}$ valence bands, and the $\Gamma_{6}, \Gamma_{7}$, and $\Gamma_{8}$ conduction bands. ${ }^{26}$ The band-gap shrinkage due to the exchange interaction among free carriers, given $b y^{27}$

$$
E_{0}(n)=\alpha N_{e}^{1 / 3},
$$

where $\alpha=2.25 \times 10^{-8} \mathrm{eV} \mathrm{cm}$ and $N_{e}$ is the electron concentration, was taken into account in the $\mathbf{k} \cdot \mathbf{p}$ calculations. For the electron effective mass, we used the room-temperature value of $0.076 m_{0}$ reported in a recent Shubnikov-de Haas study of the carrier concentration dependence of the effective mass in $n$-InP. ${ }^{28}$ The polynomial coefficients of Eq. (3.6) depend on carrier density and temperature. For instance, for InP at room temperature and a carrier density of $10^{17} \mathrm{~cm}^{-3}$, we find $A_{2}=5.009 \times 10^{4} \mathrm{meV} \AA^{2}, A_{4}=-1.523 \times 10^{6} \mathrm{meV}$ $\AA^{4}$, and $A_{6}=4.204 \times 10^{7} \mathrm{meV} \AA^{6}$, values that are not far from those reported in Ref. 5 for InP at $80 \mathrm{~K}$. The small differences in relation to the values of Ref. 5 may be attributed to the different values of the input parameters considered in the $\mathbf{k} \cdot \mathbf{p}$ model, such as the electron effective mass 
and interband matrix elements. The different temperature for which the band-dispersion parameters were calculated and the fact that the band-gap shrinkage was included in our calculations also contribute to these differences.

In our previous work on photoexcited plasmons, ${ }^{11}$ we used the calculated value $C=-0.14$ for the Faust-Henry coefficient. Since in that work, due to the range of photoexcited carrier densities only the $L^{+}$mode could be observed, and consequently only fits to the $L^{+}$peaks were possible, the choice of the Faust-Henry coefficient did not affect the results. However, in the present study we have a wider range of carrier densities that allows us to observe both the $L^{+}$and $L^{-}$coupled modes, and therefore to perform simultaneous fits to the $L^{+}$and $L^{-}$peaks. Given that the intensity ratio between these two peaks is very sensitive to the value of the Faust-Henry coefficient, in this study we have used the experimentally determined value $C=-0.46$ reported in Ref. 29. The high-frequency dielectric constant was taken as 9.61 (Ref. 30) and the phonon parameters were taken from Ref. 11.

By numerically evaluating Eq. (3.5), and using Eqs. (3.4) and (3.1), we calculate LOPCM theoretical Raman line shapes that are fitted to the Raman spectra. The line-shape model contains two adjustable parameters, namely the Fermi energy $E_{F}$ and the phenomenological electronic damping constant $\Gamma_{e}$. To determine the electron density from the fitted $E_{F}$ value, we use the relation between the carrier density and the Fermi energy in the nonparabolic conduction band, which was obtained to order $T^{7 / 2}$ by integrating an expansion of the density of states up to terms in $E^{5 / 2}$.

\section{RESULTS AND DISCUSSION}

The values of electron density in the doped layers as obtained from Hall measurements, which are listed in the fourth column of Table I, lie in the range $7 \times 10^{16}-1 \times 10^{19}$ $\mathrm{cm}^{-3}$. For the sample with the lowest doping $(A)$ the contacts were highly resistive and reliable Hall measurements were not possible. For this sample the carrier density was estimated assuming the same activation as found for sample $B$, which is the sample with the nearest doping density level. The fifth and sixth columns of Table I display the values of electron concentration and electronic damping, respectively, as determined from the LOPCM analysis of the Raman spectra that will be discussed below.

Figure 1 shows the room temperature Raman spectra of the $n$-type InP samples listed in Table I. In these spectra, we can observe several peaks whose frequency does not depend on the doping level of the sample, corresponding to the forbidden TO mode, the LO mode, and the three characteristic second-order optical peaks between 600 and $700 \mathrm{~cm}^{-1}$. ${ }^{31}$ The intensity of the LO peak, which arises from the surface depletion zone, decreases with increasing carrier concentration due to the reduction of the depletion depth and hence of the scattering volume for the unscreened LO mode. An additional peak, due to the $L^{+}$coupled modes, is observed in all the spectra throughout the whole range of the electron densities studied in this work. As can be observed in Fig. 1, the $L^{+}$modes in InP are very sensitive to changes in the electron concentration. The frequency of the $L^{+}$peak dis-

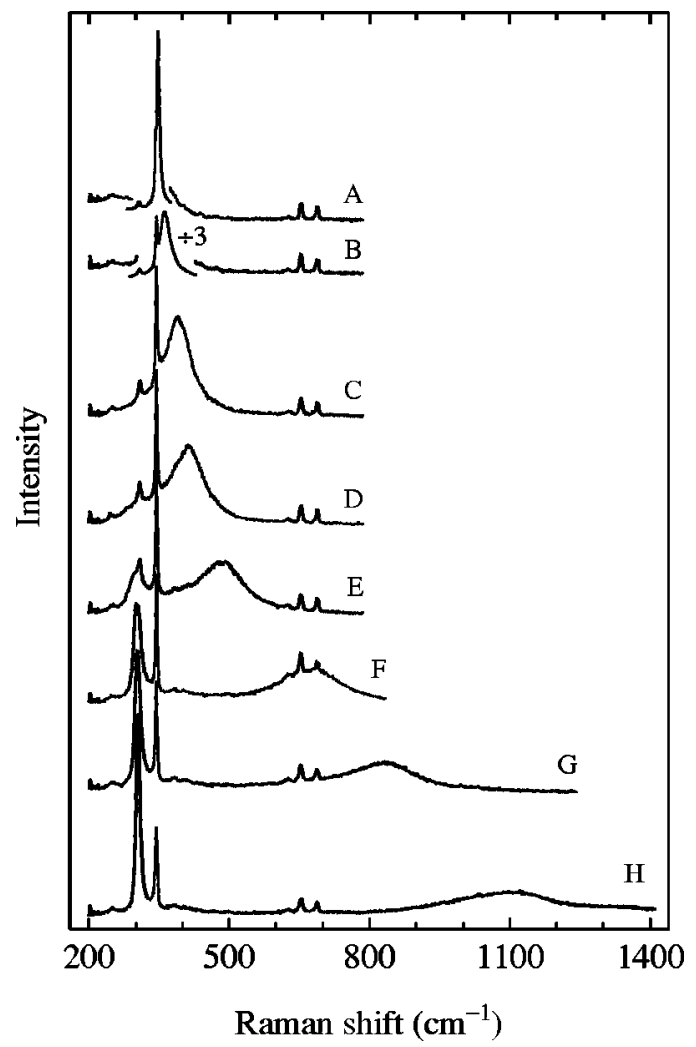

FIG. 1. Room temperature $x(y z) \bar{x}$ Raman spectra of $n$-InP samples with different doping levels. The spectra are labeled in accordance with Table I. The first-order optical region of the spectrum A was obtained using different experimental conditions (see text).

plays large shifts within the range of electron densities studied, from values very close to the LO frequency for the lowest doping sample $(A)$ up to about $1100 \mathrm{~cm}^{-1}$ for the most heavily doped sample $(H)$.

In the frequency region of the first-order optical modes, the Raman spectrum of sample $A$ shown in Fig. 1 was obtained using the triple additive configuration of the spectrometer. This allowed us to observe the depletion-zone LO modes as a shoulder on the low-frequency side of the dominant $L^{+}$peak. Since the $L^{+}$peak in spectrum $A$ was obtained using different experimental conditions, its intensity cannot be compared with the intensity of the $L^{+}$peaks in the other spectra shown in Fig. 1. The $L^{+}$coupled-mode peaks display a symmetric line shape for all doping levels, and a small width for low carrier densities. These features confirm the high degree of carrier concentration homogeneity within the probing depth of the laser beam.

The $L^{-}$coupled modes were also observed in the Raman spectra, except for the three samples with the lowest doping level $(A-C)$. In fact, for the samples in the $8.4 \times 10^{17}$ $-3.7 \times 10^{18} \mathrm{~cm}^{-3}$ range $(D-F)$, the $L^{-}$coupled modes could be resolved from the TO modes. As can be seen in Fig. $1(G, H)$, the $L^{-}$peak corresponding to the highest electron density overlaps the TO peak, giving rise to a single Raman peak close to the TO frequency, which is about six times more intense than the Raman intensity observed in the TO spectral region in undoped InP. To better resolve the $L^{-}$ coupled modes from the TO modes in samples $D-F$, we have performed Raman measurements at $80 \mathrm{~K}$ on these 


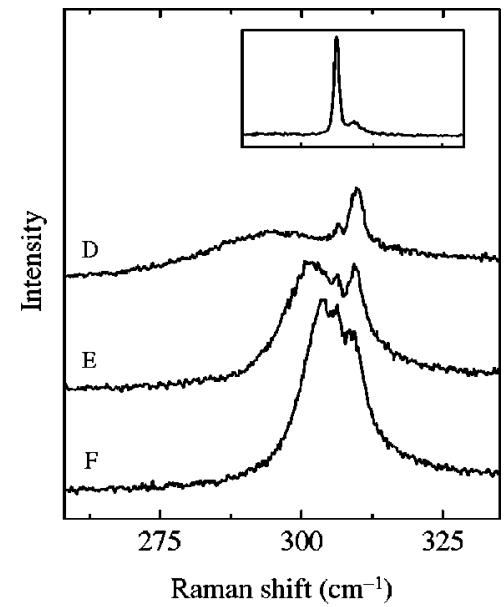

FIG. 2. High-resolution $x(y z) \bar{x}$ Raman spectra obtained at $80 \mathrm{~K}$ from samples $D, E$, and $F$ (see Table I) in the TO spectral region. Inset, Raman spectrum obtained also at $80 \mathrm{~K}$ with the sample $D$ tilted about $60^{\circ}$ off normal incidence. The frequency axes of the inset and of the main figure are coincident.

samples with high spectral resolution. Figure 2 shows the low-temperature, high-resolution Raman spectra of samples $D-F$, in which three peaks can be observed in the TO spectral region. The low-frequency peak can be unambiguously assigned to the $L^{-}$coupled modes, since its frequency depends on the carrier concentration. The other two peaks, at 306.4 and $309.4 \mathrm{~cm}^{-1}$, do not show any dependence on carrier density. The existence of an additional peak close to the TO mode, which we have also observed in undoped InP, was previously assigned to a second-order overtone of the longitudinal acoustic modes at zone edge ${ }^{32}$ lying very close to the TO frequency. Given that a precise value of the TO energy is necessary as an input of the LOPCM line-shape model, it is important to unambiguously identify the TO peak in the Raman spectra. Bearing in mind that the TO modes are forbidden in backscattering configuration from a (100) face, we have carried out low-temperature Raman measurements with the sample tilted about $60^{\circ}$ off normal incidence to circumvent the selection rules for backscattering from the (100) face. The low-temperature Raman spectrum obtained in this configuration from sample $D$ is shown in the inset of Fig. 2. The strong intensity increase of the peak at $306.4 \mathrm{~cm}^{-1}$ proves unambiguously that this peak actually corresponds to the TO mode.

Our identification of the $L^{-}$modes at frequencies very close to the TO mode differs substantially from the $L^{-}$frequencies reported by Bairamov et al. ${ }^{6}$ Although these authors do not report the laser wavelength used in their Raman measurements, the large frequency separation between the $\mathrm{L}^{-}$and the TO modes shown in Ref. 6 could only be accounted for by the use of a near-infrared laser line as excitation source. In any case, their assignment of a peak at 200 $\mathrm{cm}^{-1}$ to the $L^{-}$coupled modes for the sample in which the $\mathrm{L}^{+}$coupled modes were detected at $369 \mathrm{~cm}^{-1}$ cannot be supported by Raman line-shape calculations using the Lindhard-Mermin model. Neither the low $L^{-}$coupled-mode frequency nor the intensity ratio between the $L^{+}$and the $L^{-}$ peaks can be reproduced by the model, even for long excitation wavelengths. In Ref. 3 , the $L^{-}$coupled mode is re-

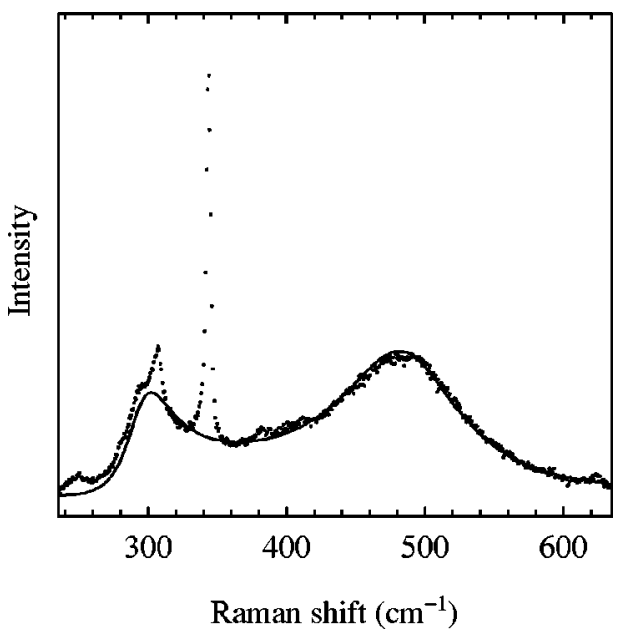

FIG. 3. Theoretical $L^{+}$and $L^{-}$line-shape fit based on the Lindhard-Mermin model (solid line) to the experimental roomtemperature Raman spectrum (dots) of sample $E$ (see Table I).

ported at $270 \mathrm{~cm}^{-1}$, a frequency much lower than those found in the present work. This is on account of the 773.8-nm wavelength used in Ref. 3, which excites coupled modes with smaller wave vectors giving rise to $L^{-}$mode frequencies well below the TO frequency. In this case, the frequencies of both $L^{+}$and $L^{-}$coupled modes are well reproduced by the Lindhard-Mermin line-shape calculations.

To determine the carrier concentration and the electronic damping for the $n$-type InP samples $A-H$ from their Raman spectra we have applied the theoretical model described in Sec. III. For samples with carrier concentration higher than $7 \times 10^{17} \mathrm{~cm}^{-3}(D-H)$ the $L^{-}$modes were detected, and the theoretical line shapes were fitted simultaneously to the $L^{+}$ and $L^{-}$experimental Raman peaks. In Fig. 3 we show the fit of the calculated $L^{+}$and $L^{-}$Raman line shapes to the experimental Raman spectrum of sample $E$. The slight difference that can be observed between the intensity of the theoretical $L^{-}$line shape and the experimental Raman spectrum could be accounted for by a slightly underestimated absolute value of the Faust-Henry coefficient and also by the fact that the TO modes and the overlapping second-order overtone discussed above are superimposed onto the $L^{-}$peak. The Raman peaks corresponding to the LO and TO modes, as well as the second-order peaks, were fitted by Lorentzian line shapes and subtracted from the Raman spectra prior to the LOPCM line-shape fit. For the samples with carrier concentration higher than $4 \times 10^{18} \mathrm{~cm}^{-3}$, in which the $L^{-}$peak completely overlaps the TO peak and the deconvolution is not possible, the Raman intensity in the TO spectral region of virgin InP was subtracted.

Raman measurements on the lowest doping sample $(A)$ at different incident powers show that the Raman spectra of this sample depend on the laser power. This is due to the generation of photoexcited carriers, ${ }^{11}$ which cannot be neglected when samples with low carrier concentration are studied. To take into account this effect, the line-shape model that we fitted to the spectrum of sample $A$ included an additional term in the electric susceptibility corresponding to the photoexcited-hole contribution. ${ }^{11}$ We have assumed the same dependence of photoexcited carrier concentrations on laser power that was found in semi-insulating InP, ${ }^{11}$ although the 
photoexcited carrier density in doped samples may be slightly smaller due to the fact that the induced defects may also act as recombination centers for the photoexcited carriers. To obtain the electron density in sample $A$ the estimated densities of photoexcited electrons were subtracted from the results of fitting the experimental spectra, and consistent values for different incident laser powers were found. The photoexcited contribution was not taken into account in previous studies reporting determinations of carrier densities in InP by means of Raman spectroscopy, and consequently, the carrier density of low-doping samples was overestimated in those studies. ${ }^{2,4-7}$ The Raman spectra of sample $B$, for which a carrier density of $3.7 \times 10^{17} \mathrm{~cm}^{-3}$ was determined, display only a very small dependence on laser power and the corresponding charge-density correction due to photoexcited carriers is not significant. Consequently, for the $n$-InP samples with higher doping levels studied in this work, photoexcited plasma corrections can be neglected.

In the fifth and sixth columns of Table I, we report the values of carrier concentration and electronic damping obtained from the LOPCM fits, in which, as already mentioned, the Fermi energy and the electronic damping are the only adjustable parameters. The values of the reduced Fermi energy $\left(E_{F} / k_{B} T\right)$ found for samples $A-H$ range from -1.35 for the lowest doping sample $(A)$, to 8.27 for the highest doping sample $(H)$. Consequently, the use of the Fermi-Dirac distribution function is necessary for a realistic description of the electron plasma through the whole range of carrier concentrations. As expected, the electronic damping increases with the doping density, but whereas for samples $A-F$ damping values are of the same order as those found for the photoexcited plasmons in semiconducting $\mathrm{InP},{ }^{11}$ the electronic damping increases considerably for the most heavily doped samples $(G, H)$. As can be seen in Table I, the carrier concentrations obtained from the LOPCM fits based on the Lindhard-Mermin model are in very good agreement with those obtained from Hall measurements, confirming that Raman-scattering can be used in a wide range of electron densities to obtain an accurate determination of the carrier density in $n$-type InP. Raman scattering provides some advantages over Hall determinations of the free-carrier densities in doped semiconductors, such as being a nondestructive, contactless technique, and not requiring an a priori knowledge of the Hall scattering factor, which depends on the electron scattering mechanisms and may differ in samples with different doping levels. ${ }^{33}$

\section{COMPARISON BETWEEN THE DRUDE AND THE HYDRODYNAMICAL MODELS AND THE LINDHARD-MERMIN MODEL}

Many of the LOPCM analyses of Raman spectra in polar semiconductors have been carried out using either a Drude model $^{12,34,35}$ or the hydrodynamical model $1^{4,10,36,37}$ for the free-carrier electric susceptibility. The computational simplicity of these two models as compared with the more involved Lindhard-Mermin calculations has favored their widespread use in the analysis of Raman spectra. In particular, in several papers dealing with Raman scattering of $n$-InP the electron concentration was determined using a Drude model ${ }^{2,3,6-8}$ or the hydrodynamical theory. ${ }^{4}$ In Ref. 7 a good agreement was found between the carrier concentrations determined by means of a Drude analysis of the Raman spectra and those obtained from Hall measurements on bulk and epitaxial $n$-InP for a wide range of carrier densities. This is a surprising result because, as it will be discussed below, our results show that the Drude model leads to significant errors in the charge-density determination, and therefore such a good agreement is not to be expected.

In this section, we assess the accuracy of the Drude and the hydrodynamical models by comparing the results of applying these models to our experimental Raman data with the results that we have obtained using the Lindhard-Mermin model. In the Drude model the electric susceptibility, which does not have spatial dispersion, is given by

$$
\chi_{e}^{(D)}(\omega)=-\frac{\epsilon_{\infty}}{4 \pi} \frac{\omega_{p}^{2}}{\omega\left(\omega+i \Gamma_{e}\right)},
$$

where $\omega_{p}=\left(4 \pi N_{e} e^{2} / \epsilon_{\infty} m^{*}\right)^{1 / 2}$ is the plasma frequency. In the hydrodynamical model an additional term is considered in the electron dynamical equation that accounts for the force arising from pressure gradients in the electron gas. ${ }^{10,38}$ Pressure gradients can be related to density gradients through the kinetic theory relation $p=N_{e} m^{*}\left\langle\mathrm{v}^{2}\right\rangle / 3$, with $\left\langle\mathrm{v}^{2}\right\rangle$ the meansquare velocity. The pressure term gives rise to spatial dispersion and the $q$-dependent electric susceptibility becomes

$$
\chi_{e}^{(H)}=-\frac{\epsilon_{\infty}}{4 \pi} \frac{\omega_{p}^{2}}{\omega^{2}-\left\langle\mathrm{v}^{2}\right\rangle q^{2}+i \omega \Gamma_{e}} .
$$

The mean-square velocity can be evaluated in terms of the Fermi integrals ${ }^{39} \mathcal{F}_{3 / 2}, \mathcal{F}_{1 / 2}$ as

$$
\left\langle\mathrm{v}^{2}\right\rangle=\frac{3 k_{B} T}{m^{*}} \frac{\mathcal{F}_{3 / 2}\left(E_{F} / k_{B} T\right)}{\mathcal{F}_{1 / 2}\left(E_{F} / k_{B} T\right)} .
$$

At room temperature, for carrier densities higher than $10^{18}$ $\mathrm{cm}^{-3}$ the mean-square velocity can be approximated by its degenerate limit $\left\langle\mathrm{v}^{2}\right\rangle \approx(3 / 5) \mathrm{v}_{F}^{2}$, whereas for lower densities the classical limit $\left\langle\mathrm{v}^{2}\right\rangle \approx 3 k_{B} T / m^{*}$ provides a better approximation.

We have used the electric susceptibility expressions Eqs. (5.1) and (5.2), corresponding to the Drude and hydrodynamical models respectively, to extract values of carrier concentration from the Raman spectra of samples $B$ to $H$, and we have compared the results with the values obtained with the Lindhard-Mermin model described in Sec. III. Sample $A$ has not been included in this analysis to avoid the problems associated with photoexcited charge. In Fig. 4 we plot the deviations of the carrier density as calculated with the Drude and the hydrodynamical models in relation to the results of the Lindhard-Mermin model for the different samples studied in this work. As generally reported in the literature, both models have been fitted only to the $L^{+}$coupled modes. As it will be discussed below, performing simultaneous fits to both $L^{+}$and $L^{-}$coupled modes would lead to additional errors.

The Drude model yields sizable differences over the whole concentration range, leading to density values that are overestimated by $\approx 30 \%$ at the lowest densities and underestimated by $\approx 20 \%$ at the highest densities. Such large differences at low-carrier densities arise because the wave- 


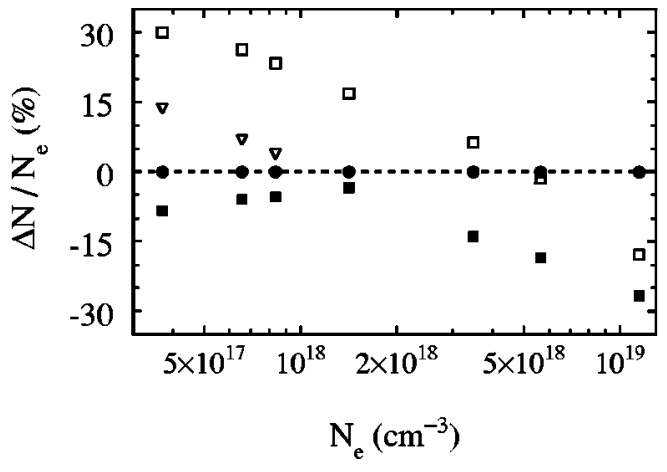

FIG. 4. Deviations of the electron densities as determined by means of the Drude model (squares), the hydrodynamical model using the classical and degenerate limits as appropriate (solid squares), and the hydrodynamical model in the degenerate limit (triangles) in relation to the results of the Lindhard-Mermin model (solid circles) for the samples studied in the present work.

vector dependence of the plasma frequency is neglected in the Drude model. Assuming parabolic conduction bands, in the limit $\left(\hbar^{2} / 2 m^{*}\right) \mathbf{k} \cdot \mathbf{q} \ll \hbar \omega$ the finite wave-vector correction to the plasma frequency can be obtained from the Lindhard-Mermin expression of the electric susceptibility [Eq. (3.4)] by expanding to the lowest order in $q / \omega$

$$
\chi_{e}^{(L M)} \approx-\frac{\epsilon_{\infty}}{4 \pi} \frac{\omega_{p}^{2}\left[1+\left\langle\mathrm{v}^{2}\right\rangle(q / \omega)^{2}\right]}{\omega(\omega+i \Gamma)} .
$$

Comparing Eqs. (5.1) and (5.4), we see that at a finite wave vector the plasma frequency increases, and therefore the Drude expression for the electric susceptibility overestimates the carrier concentration. On the other hand, for increasing carrier density the conduction-band nonparabolicity becomes more pronounced and the associated increase of the electron effective mass reduces the plasma frequency so that the parabolic-band models tend to underestimate the electron density. The nonparabolicity effects increase with the doping level and, consequently, the overestimation of the charge density caused by neglecting the plasmon dispersion is gradually compensated as the carrier concentration increases. For carrier concentrations around $6 \times 10^{18} \mathrm{~cm}^{-3}$ both models accidentally yield the same results in InP, whereas for higher carrier concentrations nonparabolicity dominates over plasmon dispersion effects.

In Fig. 4, we also show the values of carrier density obtained by fitting theoretical line shapes to the experimental Raman spectra using the hydrodynamical model in the classical or degenerate limit as appropriate. The degenerate limit in the hydrodynamical model has been customarily used regardless of the doping level, even in nondegenerate conditions. From Fig. 4 we can see that the use of the degenerate limit for samples with low-doping density leads to charge density values up to $30 \%$ higher in relation to the classical limit, and therefore it is important to consider the appropriate limit when the hydrodynamical model is applied. We observe that the carrier-density values obtained with the hydrodynamical model are below the results of the LindhardMermin model for the whole range of carrier concentrations. Although the hydrodynamical model incorporates spatial dispersion, it does not take into account the nonparabolicity of the conduction band, and hence, as discussed above, it underestimates the charge density. For carrier densities lower than $\approx 10^{18} \mathrm{~cm}^{-3}$, where the nonparabolicity effects are not important in InP, the hydrodynamical model in the classical limit is in reasonable agreement with the Lindhard-Mermin model. The validity of the hydrodynamical model can be extended up to higher carrier concentrations for other compounds such as $\mathrm{SiC}$ and $\mathrm{GaN}$ in which nonparabolicity effects are smaller. Contrary, the application of the Drude model on such compounds ${ }^{12-16}$ can yield grossly overestimated values of the carrier concentration because the error introduced by neglecting spatial dispersion is not compensated by the nonparabolicity effects.

Whereas the use of the Lindhard-Mermin model allows us to simultaneously fit the $L^{+}$and $L^{-}$coupled modes, the Drude model gives poor fits to the $L^{-}$modes. In fact, as discussed above, the Drude susceptibility [Eq. (5.1)] is formally identical to the small- $q$, high- $\omega$ limit of the LindhardMermin susceptibility [Eq. (5.4)], if $\omega_{p}^{2}$ is replaced by a wave-dependent plasma frequency $\omega_{p}^{2}(q)=\omega_{p}^{2}[1$ $\left.+\left\langle\mathrm{v}^{2}\right\rangle(q / \omega)^{2}\right]$. Since Eq. (5.4) was derived assuming $\left(\hbar^{2} / 2 m^{*}\right) \mathbf{k} \cdot \mathbf{q} \ll \hbar \omega$, neither the expansion Eq. (5.4) nor the Drude form [Eq. (5.1)] are good approximations at low frequencies.

The hydrodynamical model is often applied ${ }^{36,37}$ using an expansion of Eq. (5.2) to the lowest order in $q / \omega$ in which $\left\langle\mathrm{v}^{2}\right\rangle$ is approximated by its degenerate limit, $(3 / 5) \mathrm{v}_{F}^{2}$. This reduces the hydrodynamical electric susceptibility to the well-known expression

$$
\chi_{e}^{(H)} \approx-\frac{\epsilon_{\infty}}{4 \pi} \frac{\omega_{p}^{2}}{\omega(\omega+i \Gamma)}\left[1+\frac{3}{5} \mathrm{v}_{F}^{2}\left(\frac{q}{\omega}\right)^{2}\right],
$$

which has again the Drude form with a $q$-dependent plasma frequency. These kind of expansions, while accurate enough at high frequencies, are poor approximations at low frequencies where the $L^{-}$modes occur, and consequently, they cannot be used to model the $L^{-}$spectral region. Contrary, the use of the hydrodynamical susceptibility expression Eq. (5.2) in the line-shape models results in much better fits to the $L^{-}$ modes. Moreover, for low doping samples the $L^{+}$modes occur at low frequencies, close to the LO frequency, and therefore the fits to the $\mathrm{L}^{+}$modes are also affected by the inaccuracy of Eq. (5.5) at low energies, leading to further deviations in relation to the results of the Lindhard-Mermin model.

\section{CONCLUSION}

We have studied the LOPCM's in $n$-InP for a wide range of carrier concentrations by means of Raman spectroscopy. The $L^{+}$coupled modes have been found to be very sensitive to the free-carrier density in $n$-InP. Very good simultaneous fits to both the $L^{+}$and $L^{-}$coupled modes observed in the Raman spectra are obtained using a line-shape model based on the Lindhard-Mermin dielectric function that includes the nonparabolicity of the InP conduction band. The presence of photoexcited carriers has been taken into account in the sample with the lowest doping level. From the line-shape analysis, we obtain accurate values of the electron density in the doped samples that are in good agreement with Hall- 
effect determinations. Thus, Raman scattering provides a nondestructive, contactless means of determining the carrier concentration over a wide range of doping levels.

The line-shape theory that we have applied to determine the carrier concentration takes into account finite temperature and wave-vector effects, as well as nonparabolicity effects. Other models widely used to determine carrier concentration, such as the Drude and the hydrodynamical models, though computationally simpler, present significant departures from the Lindhard-Mermin model at different electron-density regimes. Thus, the analysis of the same Raman spectra using the Drude or the hydrodynamical model leads to significant differences in carrier concentration, up to $30 \%$ in relation to the results of the Lindhard-Mermin model, which reflect the important effects of plasmon dispersion and nonparabolicity of the conduction band on the determination of carrier density from LOPCM line shapes. These two effects act in op- posite directions; whereas neglecting plasmon dispersion leads to an overestimation of the carrier density, neglecting the nonparabolicity of the conduction band results in the underestimation of the carrier density. Thus, for a certain range of carrier densities these two effects partially compensate and the values of carrier density obtained using the Drude or the hydrodynamical model are not far from those obtained by using the Lindhard-Mermin model. Neither the Drude model nor the usual small- $q$ expression derived from the hydrodynamical model can be used to fit the $L^{-}$coupled modes because they involve poor approximations for low frequencies.

\section{ACKNOWLEDGMENT}

The authors would like to acknowledge the Spanish Ministerio de Educación y Cultura for financial support.
${ }^{1}$ See, for instance, G. Abstreiter, M. Cardona, and A. Pinczuk, in Light Scattering in Solids IV, edited by M. Cardona and G. Güntherodt, Topics in Applied Physics Vol. 54 (SpringerVerlag, Berlin, 1984), and references therein.

${ }^{2}$ V. I. Zemski, E. L. Ivchenko, D. N. Mirlin, and I. I. Reshina, Solid State Commun. 16, 221 (1975).

${ }^{3}$ D. Olego, A. Pinczuk, and A. A. Ballman, Solid State Commun. 45, 941 (1983).

${ }^{4}$ T. Nakamura and T. Katoda, J. Appl. Phys. 55, 3064 (1984).

${ }^{5}$ W. Richter, U. Nowak, H. Jürgensen, and U. Rössler, Solid State Commun. 67, 199 (1988).

${ }^{6}$ B. H. Bairamov, I. P. Ipatova, V. A. Milorava, V. V. Toporov, K. Naukkarinen, T. Tuomi, G. Irmer, and J. Monecke, Phys. Rev. B 38, 5722 (1988).

${ }^{7}$ B. Boudart, B. Prévot, and C. Schwab, Appl. Surf. Sci. 50, 295 (1991).

${ }^{8}$ D. J. Olego and H. B. Serreze, J. Appl. Phys. 58, 1979 (1985).

${ }^{9}$ S. Ernst, A. R. Goñi, K. Syassen, and M. Cardona, Phys. Rev. B 53, 1287 (1996).

${ }^{10}$ U. Nowak, W. Richter, and G. Sachs, Phys. Status Solidi B 108, 131 (1981).

${ }^{11}$ R. Cuscó, J. Ibáñez, and L. Artús, Phys. Rev. B 57, 12197 (1998).

${ }^{12}$ M. V. Klein, B. N. Ganguly, and P. J. Colwell, Phys. Rev. B 6, 2380 (1972).

${ }^{13}$ H. Yugami, S. Nakashima, A. Mitsuishi, A. Uemoto, M. Shigeta, K. Furukawa, A. Suzuki, and S. Nakajima, J. Appl. Phys. 61, 354 (1987).

${ }^{14}$ T. Kozawa, T. Kachi, H. Kano, Y. Taga, M. Hashimoto, N. Koide, and K. Manabe, J. Appl. Phys. 75, 1098 (1994).

${ }^{15}$ H. Harirna, S. Nakashima, and T. Uemura, J. Appl. Phys. 78, 1996 (1995).

${ }^{16}$ C. Wetzel, W. Walukiewicz, E. E. Haller, J. Ager III, I. Grzegory, S. Porowki, and T. Suski, Phys. Rev. B 53, 1322 (1996).

${ }^{17}$ J. P. Biersak and L. G. Haggmark, Nucl. Instrum. Methods 174, 257 (1980); J. F. Ziegler, J. P. Biersak, and U. Littmark, The Stopping and Range of Ions in Solids (Pergamon, New York, 1985).
${ }^{18}$ D. E. Aspnes and A. A. Studna, Phys. Rev. B 27, 985 (1983).

${ }^{19}$ D. T. Hon and W. L. Faust, Appl. Phys. 1, 241 (1973).

${ }^{20}$ D. Olego and M. Cardona, Phys. Rev. B 24, 7217 (1981).

${ }^{21}$ K. Wan and J. F. Young, Phys. Rev. B 41, 10772 (1990).

${ }^{22}$ J. F. Young and K. Wan, Phys. Rev. B 35, 2544 (1987).

${ }^{23}$ N. D. Mermin, Phys. Rev. B 1, 2362 (1970).

${ }^{24}$ M. Ramsteiner, J. Wagner, P. Hiesinger, K. Köhler, and U. Rössler, J. Appl. Phys. 73, 5023 (1993).

${ }^{25}$ H. R. Chandrasekhar and A. K. Ramdas, Phys. Rev. B 21, 1511 (1980).

${ }^{26}$ U. Rössler, Solid State Commun. 49, 943 (1984).

${ }^{27}$ M. Bugajski and W. Lewandowski, J. Appl. Phys. 57, 521 (1985).

${ }^{28}$ D. Schneider, D. Rürup, A. Plichta, H. U. Grubert, A. Schlachetzki, and K. Hansen, Z. Phys. B 95, 281 (1994).

${ }^{29}$ E. Anastassakis, Y. S. Raptis, M. Hünermann, W. Richter, and M. Cardona, Phys. Rev. B 38, 7702 (1988).

${ }^{30}$ O. Madelung, W. von der Osten, and U. Rössler, in Numerical Data and Functional Relationships in Science and Technology, edited by O. Madelung, Landolt-Börnstein, New Series, Group III, Vol. 17, Pt. a (Springer-Verlag, Berlin, 1982).

${ }^{31}$ L. Artús, R. Cuscó, J. M. Martin, and G. Gonzalez-Díaz, Phys. Rev. B 50, 11552 (1994).

${ }^{32}$ E. Bedel, G. Landa, R. Carles, J. P. Redoulès, and J. B. Renucci, J. Phys. C 19, 1471 (1986).

${ }^{33}$ D. Lancefield, A. R. Adams, and M. A. Fisher, J. Appl. Phys. 62, 2342 (1987)

${ }^{34}$ R. Fukasawa and S. Perkowitz, Phys. Rev. B 50, 14119 (1994).

${ }^{35}$ G. Irmer, V. V. Toporov, B. H. Bairamov, and J. Monecke, Phys. Status Solidi B 119, 595 (1983).

${ }^{36}$ P. D. Wang, M. A. Foad, C. M. Sotomayor-Torres, S. Thomas, M. Watt, R. Cheung, C. D. Wilkinson, and S. P. Beaumont, J. Appl. Phys. 71, 3754 (1992).

${ }^{37}$ H. Lee and M. V. Klein, J. Appl. Phys. 81, 1899 (1997).

${ }^{38}$ D. H. Jackson, Classical Electrodynamics (Wiley, New York, 1975).

${ }^{39}$ J. S. Blackemore, Semiconductor Statistics (Pergamon, Oxford, 1962). 